\title{
Évolution potentielle du régime des crues de la Seine sous changement climatique
}

\author{
Agnès DUCHARNE ${ }^{1}$, Eric SAUQUET ${ }^{2}$, Florence HABETS ${ }^{1}$, Michel DEQUE ${ }^{4}$, Simon GASCOIN ${ }^{1}$, \\ Ali HACHOUR ${ }^{3}$, Eric MARTIN ${ }^{4}$, Ludovic OUDIN ${ }^{1}$, Christian PAGE ${ }^{5}$, Laurent TERRAY 5 , \\ Dominique THIERY ${ }^{6}$, Pascal VIENNOT ${ }^{3}$
}

\author{
1. UMR Sisyphe, UMR 7619 CNRS/UPMC, Paris - e-mail : agnes.ducharne@upmc.fr \\ 2. Cemagref, UR Hydrologie-Hydraulique, Lyon-e-mail : eric.sauquet@cemagref.fr \\ 3. Centre de Géosciences, MINES ParisTech, Fontainebleau \\ 4. CNRM, Météo-France, Toulouse \\ 5. CERFACS, Sciences de l'Univers, URA 1875 CERFACS/CNRS, Toulouse \\ 6. BRGM, Service Eau, Orléans
}

\begin{abstract}
RÉSUMÉ. - Nous avons régionalisé 12 scénarios de changement climatique d'origine anthropique dans le bassin de la Seine, pour servir en entrée de 5 modèles hydrologiques différents. Les scénarios hydrologiques résultants s'accordent tous sur un assèchement prononcé des bassins étudiés d'ici à la fin du $21^{\text {ème }}$ siècle, avec une baisse des nappes et des débits en moyenne annuelle. A l'échelle saisonnière, la baisse des débits se répercute davantage sur les basses eaux que sur les hautes eaux, dont la baisse est moins robuste. La réponse des valeurs extrêmes est plus contrastée, et les crues extrêmes, identifiées par les quantiles décennaux QJXA10, ne changeraient pas significativement au cours du $21^{\text {ème }}$ siècle. Nos résultats suggèrent aussi que la crue centennale, extrapolée selon la méthode du gradex, resterait du même ordre de grandeur qu'actuellement.
\end{abstract}

Mots-clés : Changement climatique, régimes hydrologiques, événements extrêmes, crues, Seine

\section{Potential evolution of the Seine River flood regime under climate change}

\begin{abstract}
We regionalized 12 different scenarios of anthropogenic climate change in the Seine River basin, which were used as input to 5 different hydrological models. The resulting hydrological scenarios all agree on a marked depletion of the water resources during the $21^{\text {st }}$ century, with an annual mean decrease in both water table level and river discharge. At the seasonal scale, the reduction of river flow is more marked on low than on high flows, the decrease of which is also less robust. The response of extreme flows is even more contrasted, and the QJXA10 high-flow quantile (annual daily maximum with an average return period of 10 years) would not change significantly during the $21^{\text {st }}$ century. Our results also suggest that the 100-year flood, extrapolated using the gradex method, would remain of the same order of magnitude as today.
\end{abstract}

Key-words : Climate change, hydrological regimes, extreme events, floods, Seine River

\section{INTRODUCTION}

L'augmentation anthropique de la teneur atmosphérique en gaz à effet de serre est à l'origine très probable d'un changement du climat, appelé à s'intensifier au cours du $21^{\text {ème }}$ siècle, et dont les manifestations concernent à la fois le climat moyen et la variabilité climatique, modifiée par rapport à la variabilité naturelle [1].

Cette étude s'inscrit dans le cadre plus large du projet RExHySS [2], qui visait à estimer l'impact de ce changement climatique d'origine anthropique sur l'évolution des ressources en eau et des extrêmes hydrologiques au cours du $21^{\text {ème }}$ siècle dans deux bassins versants du Nord de la France, ceux de la Seine $\left(78600 \mathrm{~km}^{2}\right)$ et de la Somme $\left(7400 \mathrm{~km}^{2}\right)$. A l'heure actuelle, grâce au climat océanique et à l'influence régulatrice des nappes souterraines, ces hydrosystèmes soutiennent bien les différents usages de l'eau en situation normale, notamment d'un point de vue quantitatif (eau potable, prélèvements agricoles, navigation, etc.). Ils sont en revanche très vulnérables aux extrêmes hydrologiques, comme ont pu l'illustrer les crues de 1910 de la Seine et 2001 de la Somme, ou les sécheresses de 2003 et 2005.

Nous nous focaliserons ici sur l'évolution potentielle des crues dans le bassin de la Seine au $21^{\text {ème }}$ siècle, après avoir brièvement décrit les étapes préalables : construction de scénarios régionalisés du climat du $21^{\text {ème }}$ siècle, puis construction de scénarios hydrologiques, en forçant des modèles hydrologiques par ces scénarios climatiques. A toutes les étapes, nous préciserons les incertitudes associées, qui seront discutées en conclusion.

\section{SCÉNARIOS CLIMATIQUES RÉGIONALISÉS POUR LE $21^{\mathrm{e}}$ SIÈCLE}

Les premiers ingrédients d'une étude d'impact hydrologique du changement climatique sont des projections des conditions climatiques du futur, simulées par des modèles de climat global en fonction d'un scénario d'émissions anthropiques (gaz à effet de serre et aérosols) pour le $21^{\text {ème }}$ siècle. 
Une étape importante est celle de la désagrégation de ces scénarios climatiques de grande échelle (on parle aussi de régionalisation ou de descente d'échelle). En effet, si les modèles climatiques décrivent correctement les principales caractéristiques de grande échelle du climat global (anticyclones et systèmes dépressionnaires, moussons, etc.), ils en représentent assez mal les caractéristiques locales, du fait de leur faible résolution spatiale ( $>100 \mathrm{~km}$ de coté). Il s'agit donc d'introduire les hétérogénéités spatiales non résolues par les modèles climatiques de grande échelle, tout en corrigeant les biais éventuels, notamment ceux des précipitations qui sont particulièrement pénalisants pour la simulation hydrologique.

Nous avons exploité deux méthodes développées récemment par la communauté française. La première combine une désagrégation dynamique (par un modèle climatique à haute résolution) avec une correction de biais par ajustement des quantiles simulés aux quantiles observés [3]. La seconde est une méthode de désagrégation statistique basée sur l'analyse des régimes de temps [4]. On suppose que ces derniers sont bien reproduits par les modèles climatiques, ce qui permet de contraindre la recherche d'analogues entre les journées simulées et les journées observées, dont la météorologie locale remplace celle de la journée simulée dans le scénario désagrégé.

Ces méthodes sont calibrées sur le climat récent, en utilisant comme observations les données météorologiques de la base de données SAFRAN [5] sur la période 1971-2000. Nous avons testé leur validité par comparaison avec ces données, avec des résultats satisfaisants sur les régimes locaux des précipitations journalières. Les biais sont faibles, et les distributions des valeurs journalières sont assez bonnes, en intensité comme en fréquence, malgré une sous-estimation de la persistance des épisodes secs.

Sous l'hypothèse que les erreurs des modèles sont stationnaires sous changement climatique, ces méthodes permettent donc de rendre compte des changements de variabilité du climat, de l'échelle journalière à interannuelle, en plus du changement de climat moyen. Elles permettent ainsi de ne plus limiter l'impact du changement climatique aux seules ressources en eau (définies ici comme les moyennes annuelles et saisonnières des débits et niveaux des nappes, voir section III) et d'aborder les extrêmes hydrologiques (crues et étiages rares, voir section IV). Ce n'est pas le cas de la méthode plus simple des anomalies, également utilisée comme référence avec des études antérieures [e.g. 6], et qui constitue le « degré 0 » des méthodes de désagrégation.

Les incertitudes sont une question cruciale dans ce type d'études reposant sur des modèles complexes. Pour appréhender les nombreuses incertitudes liées aux scénarios désagrégés, nous avons classiquement multiplié ces derniers. Nous nous appuyons ainsi sur 12 scénarios désagrégés de changement climatique, caractérisés par 8 modèles climatiques différents, forcés par 1 ou 2 scénarios d'émissions utilisés pour les simulations du GIEC, et désagrégés par 1 à 3 méthodes différentes [2].

Ces scénarios désagrégés s'accordent sur un réchauffement au cours du $21^{\text {ème }}$ siècle $\left(\mathrm{de}+1.5\right.$ à $+3^{\circ} \mathrm{C}$ en milieu de siècle et entre +2 et $+4^{\circ} \mathrm{C}$ en fin de siècle) qui s'accompagne d'une augmentation de l'évapotranspiration potentielle. Les précipitations estivales baissent de manière systématique, avec une augmentation de la persistance des événements secs. Celle des événements pluvieux varie peu et le signe d'évolution des précipitations hivernales n'est pas certain. En revanche, les cumuls annuels baissent au cours de 21 ème siècle, de manière quasi systématique. La seule exception est une augmentation de $+2 \%$ pour un scénario de fin de siècle, et la moyenne des 12 scénarios est de $-6 \%$ et $-12 \%$, en milieu et fin de siècle respectivement.

\section{III. ÉVOLUTION DES RESSOURCES EN EAU}

L'étape suivante consiste alors à transformer ces scénarios climatiques en scénarios hydrologiques, décrivant des évolutions possibles des débits et des niveaux des nappes phréatiques dans les bassins versants. On utilise pour cela des modèles hydrologiques de bassin, qui sont d'abord calés sur les conditions actuelles, puis utilisés pour simuler le fonctionnement du bassin selon les scénarios de changement climatique désagrégés.

Pour pouvoir estimer les incertitudes associées, nous avons mobilisé 5 modèles hydrologiques (MODCOU [7], SIM [8], CLSM [9], EROS [10], GR4J [11]). Ils couvrent les principales différences entre les grandes écoles de modélisation hydrologique (hydro-météorologiques vs hydrogéologiques, distribués $v s$ globaux, à bases physiques $v s$ conceptuels) et ont tous été validés en temps présent avec de bonnes performances, en utilisant les forçages météorologiques SAFRAN. Nous avons également vérifié la capacité des combinaisons modèle de climat / méthode de régionalisation / modèle hydrologique à restituer les principales caractéristiques des débits observés. Ce point est illustré en Figure 1 par le fait que les débits observés sont dans l'écart-type des débits simulés sous scénarios temps présent par l'ensemble des modèles hydrologiques.

Nous avons ainsi construit une trentaine de scénarios hydrologiques, qui indiquent un assèchement prononcé des bassins étudiés au cours du 21 ème siècle. Il se traduit notamment par une baisse de la recharge annuelle des nappes $(-33 \%$ en fin de siècle, statistiquement significative selon le test de Student au risque $\alpha=2 \%$ en regard de la dispersion entre les différents scénarios hydrologiques). Il s'ensuit une baisse des niveaux piézométriques, qui contribue à la baisse les débits moyens. Cette dernière est robuste puisque la baisse moyenne du débit de la Seine à son exutoire à Poses $\left(-157 \mathrm{~m}^{3} / \mathrm{s}\right.$ en fin de siècle, soit $-29 \%$ du débit moyen actuel) est bien supérieure à l'incertitude, estimée par l'écart type associé $\left(76 \mathrm{~m}^{3} / \mathrm{s}\right)$. Cette baisse est déjà largement acquise à l'horizon 2050 et s'exprime sur les hautes et les basses eaux (Figure 1), avec une baisse relative maximale en étiage. L'incertitude est maximale en hautes eaux (hiver), quand les différents scénarios hydrologiques ne s'accordent pas sur le signe de l'évolution des débits. Cette incertitude est plus grande en milieu de siècle (la moitié des scénarios indiquant une augmentation en hiver) qu'en fin de siècle (seulement quatre scénarios hydrologiques indiquant une augmentation des débits mensuels de hautes eaux).

Nous nous sommes aussi attachés à hiérarchiser les différentes sources d'incertitudes, selon [12]. Nous avons ainsi montré que les incertitudes de la réponse hydrologique proviennent d'abord des modèles climatiques, puis des modèles hydrologiques et des méthodes de désagrégation avec une incertitude associée assez comparable. Ce résultat nouveau montre qu'il est important de considérer les incertitudes liées aux modèles hydrologiques et aux méthodes de désagrégation pour ne pas introduire de biais dans l'impact projeté, de même qu'il est désormais acquis qu'il ne faut pas se limiter aux scénarios issus d'un seul modèle de climat. Il faut noter ici que le modèle hydrologique CLSM a été exclu de l'analyse, en raison d'un comportement jugé incorrect dans sa 


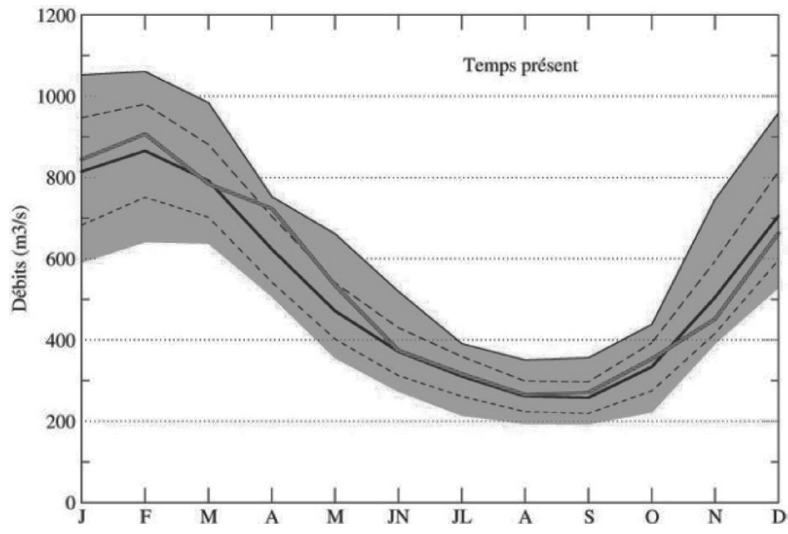

Figure 1 : Évolution des débits mensuels simulés pour la Seine à Poses par l'ensemble des modèles hydrologiques et sur l'ensemble des scénarios. L'enveloppe représente les min et max simulés, le trait épais noir la moyenne de l'ensemble, et les pointillés l'enveloppe pour un écart-type. Temps présent : la courbe rouge représente la moyenne mensuelle observée. Horizon $2050:$ la courbe bleue représente la moyenne temps présent. Horizon 2100 : les courbes bleues et roses représentent les moyennes des simulations temps présent et milieu de siècle respectivement.

réponse au changement climatique, afin de ne pas introduire d'incertitude injustifiée. Les incertitudes liées aux scénarios d'émission ou à l'échéance temporelle (milieu de siècle vs fin de siècle) sont beaucoup plus faibles que les trois premières, ce qui est cohérent avec le fait que l'essentiel des impacts est acquis dès le milieu de siècle.

\section{IV. ÉVOLUTION DU RÉGIME DES CRUES}

Nous nous sommes appuyés sur les méthodes classiques d'analyse fréquentielle pour l'hydrologie (Théorie des Valeurs Extrêmes, courbes Intensité-durée-Fréquence et débit Q-durée-Fréquence), que nous avons appliquées sur des fenêtres temporelles de 20 ans : 1980-2000 (PST pour Temps Présent) ; 2046-2065 (MS pour Milieu de Siècle) et 20812100 (FS pour Fin de Siècle). L'extension de ces fenêtres est conditionnée par la disponibilité des sorties des simulations climatiques. Nous avons fait l'hypothèse de pseudo-stationnarité des séries sur ces séquences temporelles courtes (les tendances induites par les évolutions du climat sur 20 ans ne sont pas suffisamment appuyées), ce qui autorise l'usage des statistiques classiques. Nous séparons dans la suite l'analyse des pointes de crues décennales (i.e. valeurs ayant une probabilité 0.1 d'être dépassée chaque année), qui peuvent être caractérisées avec confiance sur ces périodes, de celle des crues centennales (i.e. valeurs ayant une probabilité 0.01 d'être dépassée chaque année), pour lesquelles la part de l'extrapolation est beaucoup plus importante.

\section{IV.1. Crues décennales dans le basin de la Seine}

Les variables retenues pour décrire l'évolution de ces crues sont (i) le quantile décennal des débits maximum journaliers, QJXA10, obtenu par ajustement de l'échantillon des

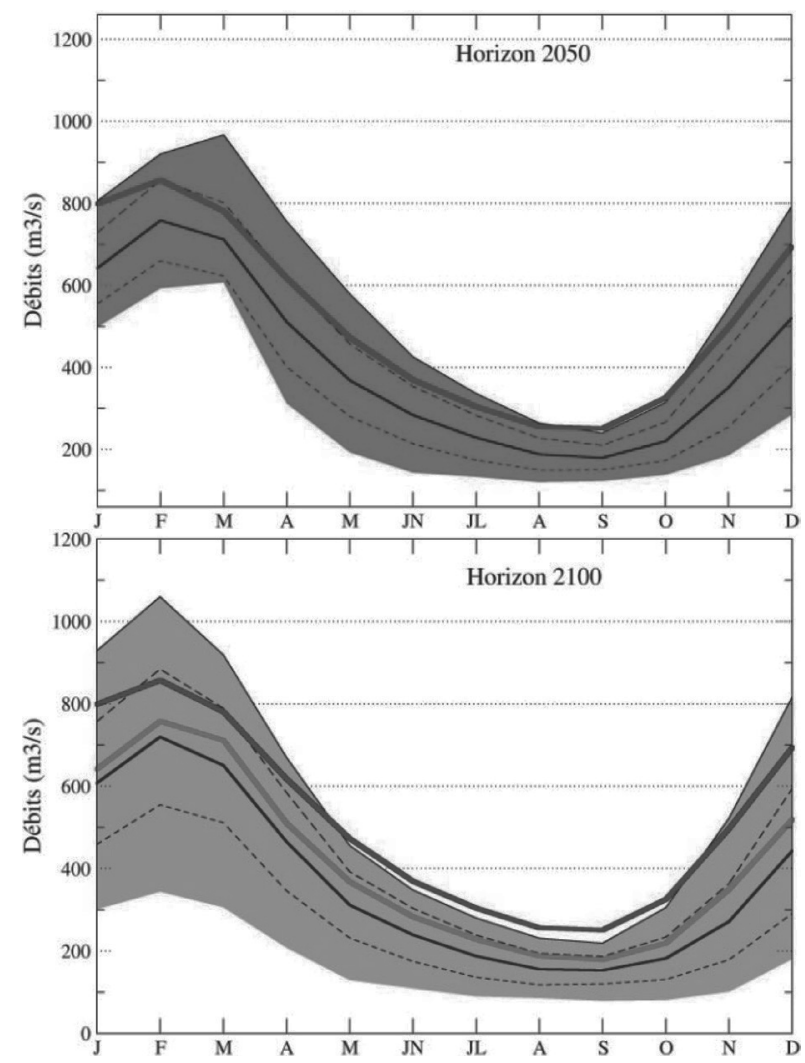

maxima journaliers annuels à la loi de Gumbel, (ii) la durée caractéristique de crue $D$, estimée sur une forme de crue moyenne [13]. Ces variables ont été sélectionnées pour leur pertinence pour la gestion et la facilité de leur caractérisation automatique sur un grand nombre de chroniques, sachant que les débits ont été simulés en 154 stations du bassin de la Seine par au moins un modèle hydrologique. Il faut cependant noter que seules 8 stations (dont la Seine à Paris et à Poses) ont été traitées par les 5 modèles.

Le principal résultat est l'absence d'évolution significative du régime des crues dans le bassin de la Seine, les changements simulés étant modérés sans cohérence de signe. Les quantiles $Q J X A 10$ évoluent entre -10 et $+10 \%$ en milieu de siècle, ces évolutions étant d'un ordre de grandeur inférieur ou égal à l'incertitude actuelle. En effet, l'intervalle de confiance à $95 \%$ autour du QJXA10 actuel, du seul fait des fluctuations d'échantillonnage, est entre $-12 \%$ et $+25 \%$ de la valeur centrale (valeurs médianes des bornes de l'intervalle de confiance sur les stations exploitées). En fin de siècle, le nombre de stations avec des diminutions du QJXA10 est plus important qu'en milieu de siècle, mais la dispersion et les incertitudes restent élevées. La durée caractéristique de crue $D$ varie peu sous changement climatique (Figure 2). On peut noter qu'au contraire, la diminution des étiages sévères est systématique sur l'ensemble des stations considérées [2].

Ces résultats sont notamment illustrés sur la Seine à Paris, station intégratrice des modifications amont, qui montre des variations modérées des débits de crue au fil du $21^{\text {ème }}$ siècle (Figure 3). Une légère réduction du QJXA10 est perceptible en fin de siècle, où la médiane des crues décennales correspond aux crues ayant actuellement une période entre 3 et 8 ans, en fonction des différents modèles hydrologiques. En parallèle, la valeur actuelle de $Q J X A 10$ serait donc plus rarement dépassée en fin de siècle. 


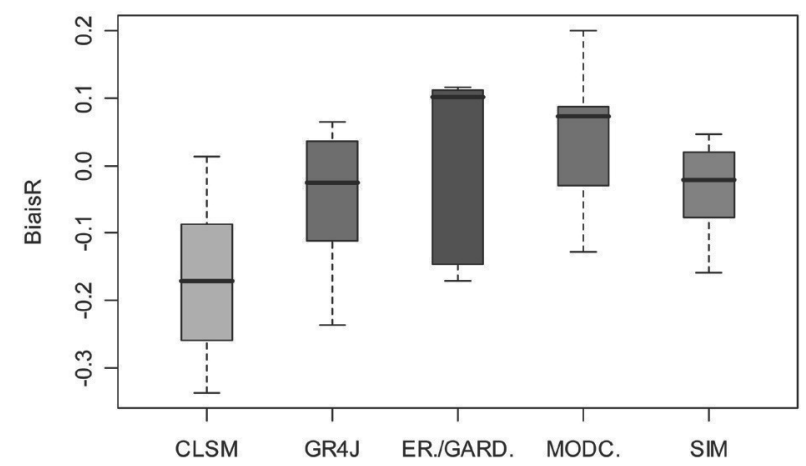

QJXA10

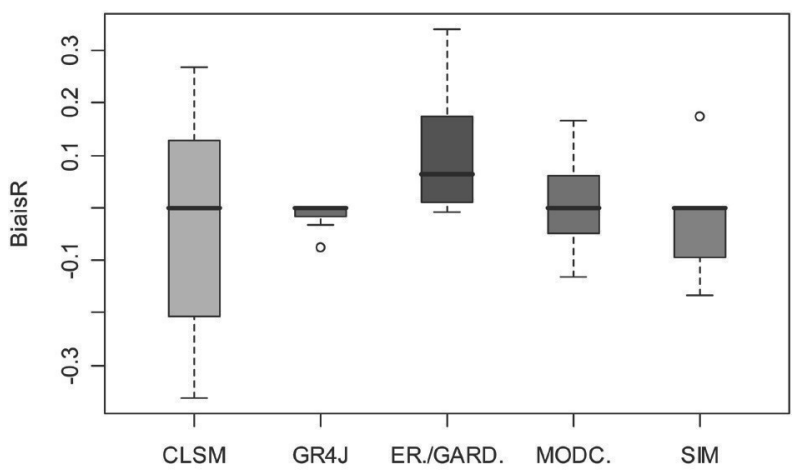

Durée de crue D

Figure 2 : Écart relatif médian entre les valeurs temps présent et milieu de siècle des variables retenues pour caractériser des crues dans l'ensemble des stations du domaine RExHySS (Seine et Somme).

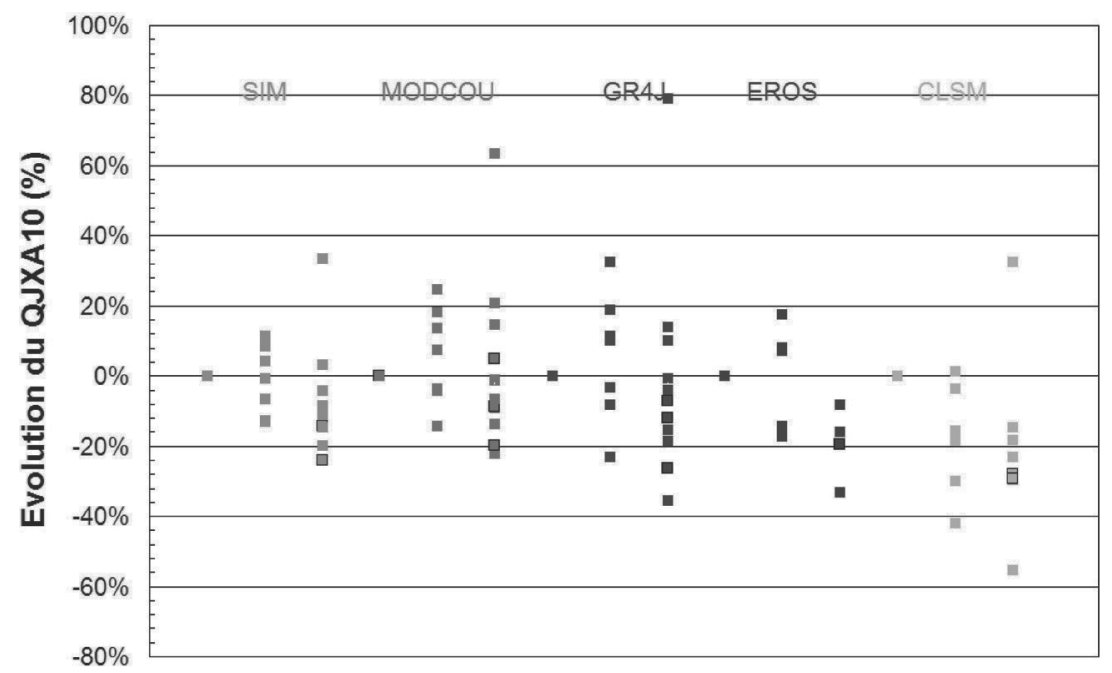

Figure 3 : Évolution du QJXA10 simulé pour la Seine à Paris. Les évolutions sont calculées par rapport aux débits simulés sous scénarios désagrégés temps présent. Pour chaque scénario, la première valeur correspond aux résultats temps présent ( $0 \%$ de changement relatif quel que soit le scénario), la deuxième (lorsqu'elle existe) au milieu de siècle, et la troisième à la fin de siècle. Les points entourés en noir correspondent aux scénarios A2.

\section{IV.2. Crues centennales à Paris}

Nous avons repris la méthode d'extrapolation des quantiles de crue rares utilisée par l'Institution Interdépartementale des Barrages-Réservoirs du Bassin de la Seine [14], conditionnées par le gradex des précipitations. Le principe repose sur l'hypothèse selon laquelle les crues les plus rares ont lieu quand le bassin contributif approche de la saturation, si bien que « tout accroissement de précipitation $\mathrm{dR}$ entraîne un accroissement dQ du débit qui tend à devenir égal à $\mathrm{dR}$ » [15]. Pour les valeurs les plus rares, la distribution des débits de crue s'écarte alors de la distribution des valeurs extrêmes ajustée aux crues observées (loi de Gumbel) pour tendre vers une asymptote parallèle à la distribution des valeurs extrêmes de la précipitation, dont la pente est nommée gradex.

Cette méthode nécessite l'examen conjoint des pluies et des débits extrêmes (saisonnalité et intensité) sur des durées cohérentes avec la dynamique de crue. Dans cette application ciblée sur la Seine à Paris, nous avons analysé les cumuls de pluie et les débits moyens sur des durées de 1 à 24 jours. Les quantiles de précipitation (Figure 4) ont été déduits de l'ajustement de la loi exponentielle sur des échantillons extraits selon l'approche sup-seuil. Pour les débits, nous avons ajusté la loi de Gumbel aux échantillons de maxima annuels, et appliqué le modèle convergent QdF [16] pour assurer une cohérence entre quantiles de débits moyens. Selon [14], l'extrapolation gradex a reposé sur la durée de 24 jours, la plus cohérente avec la dynamique de crue dans ce bassin versant de $43800 \mathrm{~km}$, et elle s'est limitée aux périodes de retour $T \geq 50$ ans. Toujours selon [14], les quantiles des débits moyens sur des durées plus courtes, dont le QJXA100 (quantile centennal des débits maximum journaliers), ont enfin été déduits des quantiles du maximum de débit moyen sur 24 jours $(V C X 24 J)$ sur la base de la forme de l'hydrogramme à Paris.

Les Figure 4 et Figure 5 illustrent l'évolution des quantiles décennaux de précipitation et des quantiles centennaux des débits, telle que simulée entre la période temps présent (PST) et la fin du $21^{\text {ème }}$ siècle (FS). Elles permettent d'abord de comparer les quantiles simulés pour le temps présent avec les quantiles déduits des observations, représentés par la droite verticale rouge. Cette analyse suggère que les scénarios régionalisés, bien qu'ils restituent de manière fiable la distribution moyenne des pluies (section II), ont tendance à sous-estimer les maxima de précipitation, y compris en cumul sur plusieurs jours. Les scénarios hydrologiques 
(combinaisons scénarios régionalisés / modèles hydrologiques) montrent également des biais sur les maxima du débit, mais leur signe n'est pas systématique, même si la sousestimation est plus fréquente. Ces biais sont imputables à l'imperfection des forçages climatiques simulés (y compris les précipitations extrêmes) et à celle de la transformation pluie-débit. Ceci illustre la difficulté à simuler la variabilité hydro-météorologique actuelle. De ce fait, les quantiles fin de siècle ne peuvent s'interpréter que relativement aux quantiles temps présent des scénarios hydrologiques associés, et ne doivent surtout pas être comparés avec les quantiles déduits des observations actuelles.

En ce qui concerne l'impact du changement climatique sur ces quantiles, le principal résultat est l'absence de tendance systématique sur les crues centennales, puisque les points sur la Figure 5 sont dispersés des deux cotés de la première bissectrice, qu'il s'agisse des pointes de crue journalières, ou des débits moyens sur 24 jours. Pour le quantile journalier centennal $Q J X A 100$, la moitié seulement des scénarios hydrologiques conduisent à des évolutions significatives, toujours sans cohérence de signe (24 points non significatifs entre les droites enveloppes, contre 11 au-dessus et 13 en dessous). Pour le quantile centennal de débit moyen sur 24 jours, $\operatorname{VCX} 24 J(T=100$ ans $)$, les tendances à la baisse sont en revanche plus nombreuses ; la différence de comportement est attribuable au gradex des pluies qui, pour certains scénarios climatiques, induit un infléchissement plus fort après le point pivot pour l'extrapolation placé à $T=50$ ans.

Il faut noter que les évolutions les plus fortement positives sur ces quantiles centennaux des débits sont principalement associées à un scénario désagrégé, déduit du modèle climatique CCCMA. Ce scénario (non simulé par le modèle EROS) entraîne une augmentation de plus de $50 \%$ des quantiles centennaux, ce qui s'explique sans doute par sa forte augmentation du quantile décennal du P12JXA (+30\% ; Figure 4).

Enfin, les extrêmes des précipitations et des débits ne montrent pas de différences fortes en fin de siècle selon les scénarios d'émission A1B ou A2, comme l'illustre la Figure 4 pour les pluies extrêmes. Ce résultat, qui peut s'expliquer par le faible nombre de projections A2, est cohérent avec la hiérarchisation des incertitudes reportée en section III.

\section{CONCLUSIONS}

Les résultats de cette étude peuvent se résumer par des variations probablement modérées du régime des crues, qu'il s'agisse des crues décennales comme des crues centennales. Ce résultat, beaucoup moins inquiétant que la baisse générale ressources en eau projetée en parallèle [2], doit cependant être regardé avec beaucoup de précautions, vues les incertitudes fortes qui l'entachent.

Une manière d'approfondir cette question est de comparer ces résultats avec ceux d'autres études dans des bassins européens proches de celui de la Seine. Nous avons abouti à la même conclusion de changement non significatif des crues dans le bassin de la Somme [2], comme d'autres auteurs dans des bassins belges [17], dans le bassin de la Loire [18], et comme une étude antérieure dans le bassin de
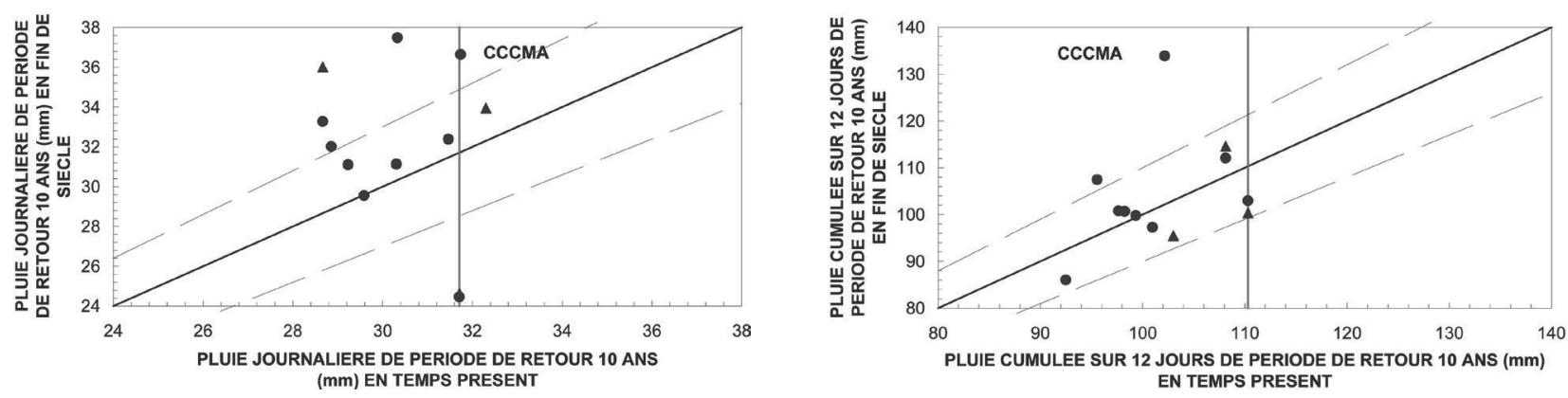

Figure 4 : Évolution des quantiles décennaux de la précipitation moyenne à l'amont de Paris entre le climat présent (PST) et la fin du siècle (FS) : maximum journalier annuel PJXA10 et maximum annuel du cumul sur 12 jours P12JXA $(T=10$ ans). Un point par scénario climatique régionalisé $(\bullet:$ scénarios régionalisés A1B; $\mathbf{\Delta}:$ scénarios régionalisés $A 2$ - issus du modèle climatique ARPEGE). La droite oblique est la première bissectrice et celles en tireté définissent les enveloppes à $\pm 10 \%$ des valeurs PST. La droite verticale rouge donne les quantiles décennaux déduits des précipitations SAFRAN pour la période PST.
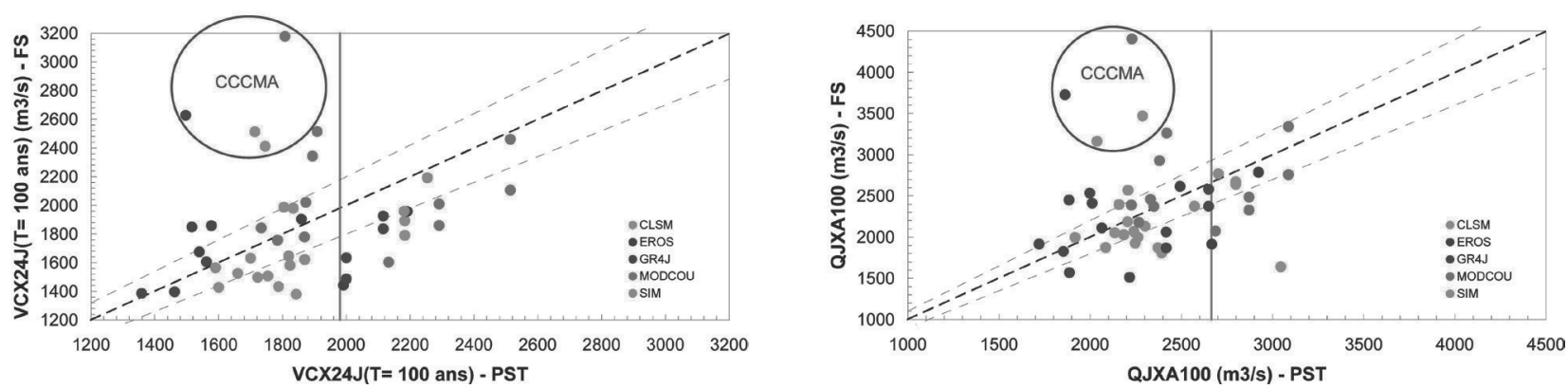

Figure 5 : Évolution des quantiles centennaux du débit à Paris entre le climat présent (PST) et la fin du siècle (FS) : débits moyens sur 24 jours $\operatorname{VCX} 24 J(T=100$ ans) et débits journaliers QJXA100. Un point par scénario hydrologique. La droite en tireté est la première bissectrice et celles en pointillé définissent les enveloppes à $\pm 10 \%$ des valeurs PST. La droite verticale rouge donne les quantiles centennaux déduits des observations de débit [14]. 
la Seine [6]. Au contraire, [19] montrent une forte augmentation des crues centennales dans le Nord de la France, mais cette étude à l'échelle européenne ne s'appuie que sur un unique scénario climatique, sans correction de biais, ce qui limite considérablement la portée de ce résultat en regard des incertitudes révélées par les autres études.

La comparaison de ces différentes études suggère aussi que la réponse des débits de crue est étroitement liée à la réponse de précipitations hivernales, avec une forte sensibilité (des variations faibles des précipitations entraînant des variations relativement plus importantes des débits, ce que confirme la réponse au scénario désagrégé issu du modèle climatique CCCMA).

Malheureusement, l'évolution des précipitations est certainement l'élément le plus incertain des projections du changement climatique. La dispersion des projections est importante, à quoi se rajoute une dispersion liée aux méthodes de désagrégation. L'incertitude totale reste pour l'instant importante, et interdit d'utiliser les projections hydrologiques comme " prévision ». La baisse des précipitations annuelles que nous anticipons dans le bassin de la Seine (comme dans celui de la Somme) représente cependant la meilleure projection actuellement disponible, car convergente pour de nombreux scénarios de changement climatique correspondant à l'état de l'art [1], et pour deux méthodes de désagrégation tout à la fois très différentes dans leur principe, et validées en climat récent sur le domaine.

Notons enfin que cette étude, comme les autres études citées, se limite à l'impact direct du changement climatique anthropique sur le régime hydrologique, et néglige les autres facteurs d'évolution possibles, comme les changements d'occupation des terres ou d'aménagement / gestion des cours d'eau.

\section{REMERCIEMENTS}

Ce travail a été réalisé dans le cadre du projet RExHySS (Impact du changement climatique sur les Ressources en eau et les Extrêmes Hydrologiques dans les bassins de la Seine

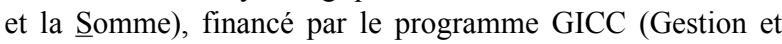
Impact du Changement Climatique) du MEEDDM.

\section{RÉFÉRENCES ET CITATIONS}

[1] IPCC, Working Group I (2007) - Climate change 2007 : the physical science basis, 4th Assessment Report, Genève

[2] Ducharne A., Habets F., Deque M., EvauX L., Hachour A., LEPAILliER A., LEPELLETIER T., MARTIN E., OUDIN L., PAge C., Ribstein P., SAuquet E., Thiery D., TERray L., VIENNOT P., BOE J., BOURQUi M., CRESPI O., GASCOIN S., RIEU J. (2009) - Impact du changement climatique sur les Ressources en eau et les Extrêmes Hydrologiques dans les bassins de la Seine et la Somme. http ://www.sisyphe.jussieu. $\mathrm{fr} / \sim$ agnes/rexhyss/documents rapport.php, Rapport final $d u$ projet RExHySS, Programme GICC. $62 \mathrm{pp}$

[3] Déqué M. (2007) — Frequency of precipitation and temperature extremes over France in an anthropogenic scenario : Model results and statistical correction according to observed values. doi10.1016/j.gloplacha.2006.11. 030, Global and Planetary Change. 57 16-26

[4] Boé J., Terray L., Habets F., \& Martin E. (2006) - A simple statistical-dynamical downscaling scheme based on weather types and conditional resampling, J. Geophys. Res . $111 \mathrm{D}$ 23106
[5] Quintana-Segui P., Le Moigne P., Durand Y., Martin E., Habets F., Baillon M., Franchisteguy L., Morel S., \& NoILhan J.(2008) - The SAFRAN atmospheric analysis : Description and validation, J. Applied Meteorol. and Climatology. 47 92-107

[6] Ducharne A., Baubion C., BeAudoin N., Benoit M., Billen G., Brisson N., GARnier J., KieKen H., Lebonvallet S., Ledoux E., Mary B., Mignolet C., POUX X., SAubOuA E., SCHOTT C., THÉRY S., \& VIENNOT P. (2007) - Long term prospective of the Seine river system : Confronting climatic and direct anthropogenic changes. doi10.1016/j.scitotenv.2006.12.011, Science of the Total Environment. 375 292-311

[7] Ledoux E., Gomez E., Monget J.M., Viavattene C., Viennot P., Ducharne A., Benoit M., Mignolet C., Schott C., \& Mary B. (2007) - Agriculture and Groundwater Nitrate Contamination in the Seine Basin. The STICS-MODCOU modelling chain. doi10.1016/j.scitotenv.2006.12.002, Science of the Total Environment. 375 33-47

[8] Habets F., Boone A., Champeaux J.L, Etchevers P., FRANCHISTEGUY L., LEBlois E., LEDOUx E., LE MOIGNE P., Martin E., MOREl S., NOILHAN J., QUINTANA-SEGUI P., Rousset-RegimbeaU, F, \& VIENNOT P. (2008) - The SAFRAN-ISBA-MODCOU hydrometeorological model applied over France, Journal of Geophysical Research (D : Atmospheres), 113 D06113(2008)18

[9] Gascoin S., Ducharne A., Ribstein P., Carli M., \& Habets F. (2009) - Adaptation of a catchment-based land surface model to the hydrogeological setting of the Somme River basin (France). doi10.1016/j.jhydrol.2009.01.039, J. Hydrol. 368(1-4) 105-116

[10] Thiery D., \& Moutzopoulos C. (1995) — Un modèle hydrologique spatialisé pour la simulation de très grands bassins : le modèle EROS formé de grappes de modèles globaux élémentaires. In VIII ${ }^{e s}$ journées hydrologiques de l'ORSTOM "Régionalisation en hydrologie, application au développement ", Le Barbé et E. Servat (Eds.), ORSTOM Editions. 285-295

[11] Perrin C., Michel C., \& Andréassian V. (2003) - Improvement of a parsimonious model for streamflow simulation, Journal of Hydrology. 279(1-4) 275-289

[12] DÉQUÉ M., ROWELL D.P., LÜTHI D., GIORGI F., CHRISTENSEN J.H., ROCKEL B., JACOB D., KJELlSTRÖM E., DE CASTRO M., \& VAN DEN HURK B. (2007) - An intercomparison of regional climate simulations for Europe : assessing uncertainties in model projections. doi10.1007/s10584-006-9228-x, Clim. Change

[13] Sauquet E., Ramos M.H., Chapel L., \& Bernardara P. (2008) - Stream flow scaling properties : investigating characteristic scales from different statistical approaches. DOI : $10.1002 /$ hy p. 6952 \& Erratum DOI : 10.1002/hyp.7192, Hydrol. Processes. 22(17) $3462-3475$

[14] I.I.B.R.B.S. (2006) - Etude des phénomènes météorologiques océaniques et méditerranéens, interagissant sur la génération des crues exceptionnelles du bassin de la Seine amont de Paris, Rapport final de synthèse. 92pp

[15] Guillot P., Duband D. (1967) — La méthode du Gradex pour le calcul de la probabilité des crues à partir des pluies. Colloque International sur les crues et leur évaluation, Leningrad, 15-22 Août 1967, IASH publication. 84 560-569

[16] Sauquet E., Javelle P., Le Clerc S. (2003) - Description des régimes hydrologiques des hautes eaux : nouvelle formulation pour l'analyse en débit-durée-fréquence et applications en ingénierie, Ingénieries EAT. 34 3-16

[17] GODERNIAUX P., BROUYÈRE S., FOWLER H. J., BLENKINSOP S., TherRien R., ORBAN P., \& DASSARgueS A. (2009) Large scale surface-subsurface hydrological model to assess 
climate change impacts on groundwater reserves. doi10.1016/j. jhydrol.2009.04.017, J. Hydrol. 373 122-138

[18] Moatar F., Ducharne A., Thiery D., Bustillo V., Sauquet E., \& VIDAL J.-P. (2010) - La Loire à l'épreuve du changement climatique, Géosciences. 12 78-87
[19] Dankers R., \& Feyen L. (2008) - Climate change impact on flood hazard in Europe : An assessment based on highresolution climate simulations. doi :10.1029/2007JD009719, J. Geophys. Res. 113 D19105 